\title{
O odontologista frente a prevenção da Pneumonia Associada a Ventilação Mecânica (PAVM) na Unidade de Terapia Intensiva (UTI)
}

\author{
The dentist facing the prevention of Pneumonia Associated with Mechanical Ventilation (PAVM) in \\ the Intensive Care Unit (ICU)
}

El odontólogo afronta la prevención de la Neumonía Asociada a la Ventilación Mecánica (MVAP) en la Unidad de Cuidados Intensivos (UCI)

\author{
Bruna Elizabete Rocha Nery da Costa \\ ORCID: https://orcid.org/0000-0001-7853-7420 \\ Centro Universitário Fametro, Brasil \\ E-mail: nerybruna07@gmail.com \\ Deille Lopes da Silva \\ ORCID: https://orcid.org/0000-0001-8505-0653 \\ Centro Universitário Fametro, Brasil \\ E-mail: odontodeilllelopes@gmail.com \\ Livia Coutinho Varejão \\ ORCID: https://orcid.org/0000-0001-6789-4203 \\ Especialista em pacientes com necessidades especiais, Brasil \\ E-mail: draliviacoutinho@gmail.com
}

\begin{abstract}
Resumo
Introdução: a PAVM é considerada uma das principais infecções relacionadas à assistência à saúde com índices cada vez mais elevados em uma UTI, esses números podem variar de $9 \%$ a $67 \%$ de todos os pacientes em uso prolongado ou não, da ventilação mecânica. Objetivo Geral: conhecer os desafios enfrentados pelo odontologista na prevenção da PAVM em uma UTI. Metodologia: Para a realização desse estudo optou-se por uma pesquisa na modalidade de revisão integrativa de literatura. Resultados e Discussão: a integração do cirurgião-dentista na equipe multiprofissional atuante nas UTIs leva a ampliação do campo de ensino e atuação do profissional, possibilitando a formulação e adaptação de diretrizes e bundles, pois são os profissionais mais capacitados nessa área de conhecimento. Considerações Finais: Toda equipe multidisciplinar em saúde alocada nas UTIS's, inclusive os odontologistas, devem agir em concordância, adotando medidas de prevenção da PAVM a fim de reduzir os riscos para a suas ocorrências, sempre seguindo uma linha de cuidado integrada, diminuindo assim, a taxa de mortalidade de seus pacientes.
\end{abstract}

Palavras-chave: Cirurgião-dentista; Pneumonia associada a ventilação mecânica; Prevenção.

\begin{abstract}
Introduction: VAP is considered one of the main infections related to health care with increasingly higher rates in an ICU, these numbers can vary from $9 \%$ to $67 \%$ of all patients on prolonged or non-extended use of mechanical ventilation. General Objective: to know the challenges faced by dentists in preventing VAP in an ICU. Methodology: To carry out this study, we opted for a research in the modality of integrative literature review. Results and Discussion: the integration of the dentist in the multidisciplinary team working in the ICUs leads to the expansion of the professional's teaching and performance field, enabling the formulation and adaptation of guidelines and bundles, as they are the most qualified professionals in this area of knowledge. Final Considerations: The entire multidisciplinary health team allocated to the ICU's, including dentists, must act in accordance, adopting measures to prevent VAP in order to reduce the risks for its occurrence, always following an integrated line of care, thus reducing, the death rate of their patients.
\end{abstract}

Keywords: Dental surgeon; Ventilator-associated pneumonia; Prevention.

\section{Resumen}

Introducción: la NAV es considerada una de las principales infecciones relacionadas con la asistencia sanitaria con tasas cada vez más elevadas en una UCI, estas cifras pueden variar del $9 \%$ al $67 \%$ de todos los pacientes en uso prolongado o no prolongado de ventilación mecánica. Objetivo general: conocer los desafíos que enfrentan los odontólogos en la prevención de NAV en una UCI. Metodología: Para la realización de este estudio se optó por una investigación en la modalidad de revisión integradora de la literatura. Resultados y Discusión: la integración del odontólogo en el equipo multidisciplinario que trabaja en las UCIs conlleva la ampliación del campo docente y de 
desempeño del profesional, posibilitando la formulación y adecuación de guías y paquetes, por ser los profesionales más calificados en esta área de Conocimiento. Consideraciones finales: Todos los equipos de salud multidisciplinarios adscritos a las UCI, incluidos los odontólogos, deben actuar en consonancia, adoptando medidas de prevención de la NAV con el fin de reducir los riesgos de su ocurrencia, siguiendo siempre una línea de atención integral, reduciendo así la tasa de mortalidad de sus pacientes.

Palabras clave: Cirujano dentista; Neumonía asociada a ventilación mecánica; Prevención.

\section{Introdução}

A pneumonia é caracterizada por um processo inflamatório que acomete o parênquima pulmonar, e que afeta os brônquios, bronquíolos, alvéolos e o interstício, os quais ficam repletos de exsudatos com características purulentas, acarretando assim, em uma disfunção na hematose, levando a um quadro de insuficiência respiratória rápida e progressiva e ocasionando sintomas como fortes dores torácicas, fadiga e desconforto respiratório (Barcellos et al., 2020).

Compreende-se por Intubação a inserção de um tubo no percurso da traqueia, através da cavidade oral ou do nariz, que está conectado ao um ventilador mecânico. Seu principal indicador ocorre em situações em que há danos na manutenção da permeabilidade das vias aéreas, nesses casos, este é considerado como um dos principais procedimentos que podem reestabelecer o quadro crítico de saúde dos pacientes com o estado de saúde grave (Branco et al., 2020).

Neste seguimento, a ventilação mecânica é um recurso de suporte avançado de vida utilizado em uma UTI, que visa substituir a respiração espontânea através de ventilador mecânico, que é acoplado ao paciente por via artificial, este pode ocorrer através de um tubo orotraqueal (TOT) ou traqueostomia (TQT) (Santos et al., 2020).

Segundo, Pinto et al., (2021) a PAVM é considerada uma das principais infecções relacionadas à assistência à saúde (IRAS) com índices cada vez mais elevados em uma UTI, esses números podem variar de 9\% a 67\% de todos os pacientes em uso prolongado ou não, da ventilação mecânica (VM) (Pinto et al., 2021). Sua ocorrência contribui para o aumento da mortalidade dos pacientes, e tem como principal origem a aspirativa, tendo como a principal fonte as secreções das vias áreas superiores seguidas da inoculação exógena de material contaminado ou do refluxo do trato gastrintestinal por meio da IOT.

Desta forma, nota-se a necessidade da adoção de medidas de prevenção da PAVM em uma UTI. Nesse sentido, a odontologia hospitalar traz ao paciente a melhora do quadro sistêmico com a manutenção da saúde bucal, visando o tratamento global do mesmo (Oliveira et al., 2021). Como reforço a esta aplicabilidade, a Lei no 2.776/2008 que foi aprovada no Senado Federal, estabelece a obrigatoriedade da presença de profissionais de Odontologia na UTI (Alecrim et al., 2019).

Sendo assim, frisa-se, portanto, a necessidade de o odontologista integrar a equipe multidisciplinar dos hospitais, a fim de atender os pacientes com enfermidades que impossibilitem, por algum motivo, a auto higiene. O cirurgião-dentista especialista em odontologia hospitalar é o profissional mais capacitado a fazer o controle de microrganismos na cavidade oral nesses casos (Furtado et al., 2020).

Segundo Silva et al. (2021), os pacientes internados em âmbito hospitalar, principalmente nas UTI's, encontram-se impossibilitados de manter uma adequada higienização bucal, necessitando do auxílio de profissionais da saúde para esta atividade. Assim, cabe ao Cirurgião-Dentista realizar um minucioso exame clínico, tratar lesões bucais e ainda realizar planos de cuidados que contemplem a necessidade daquele paciente, para permitir que o tratamento médico não seja interrompido por situações secundarias e que o paciente se recupere rapidamente.

Com isso, nota-se que a presença do cirurgião-dentista na UTI é imprescindível para a prevenção, localização e erradicação de possíveis focos infecciosos nos pacientes internados, sendo enfatizado que a presença do dentista integrado à equipe multidisciplinar leva à redução da mortalidade nas UTI's, e que este se dá principalmente pela elaboração e aplicabilidade de novos protocolos de biossegurança por esses profissionais, que abrangem diversos aspectos como o uso adequado de equipamentos de proteção individual e recomendações para o atendimento odontológico, a fim de diminuir os riscos de propagação do vírus (Oliveira et al., 2021). 
Nesse seguimento, o objetivo geral desse estudo é conhecer os desafios enfrentados pelo odontologista na prevenção da PAVM em uma UTI.

\section{Metodologia}

Para a realização desse estudo optou-se por uma pesquisa na modalidade de revisão integrativa de literatura. A revisão integrativa determina o conhecimento atual sobre uma temática específica, já que é conduzida de modo a identificar, analisar e sintetizar resultados de estudos independentes sobre o mesmo assunto (Mendes, Silveira \& Galvão, 2008).

A Revisão Integrativa é um método de pesquisa apontado como ferramenta de grande relevância no campo da saúde, por proporcionar a busca, a avaliação crítica e a síntese de evidências sobre um tema investigado. Esses aspectos facilitam a identificação dos resultados relevantes, de lacunas que direcionam para o desenvolvimento de futuras pesquisas e auxiliam o profissional a escolher condutas e a tomar decisões, proporcionando um saber crítico (Souza, Silva \& Carvalho, 2010).

A coleta das informações para a pesquisa bibliográfica será por meio da exploração da base de dados da Biblioteca Virtual em Saúde (BVS), Biblioteca Cientifica Eletrônica Virtual (SCIELO) e Literatura Latino - Americana e do Caribe em Ciências da Saúde (LILACS), e Bibliografia Brasileira de Odontologia (BBO).

A busca na base de dados foi orientada pelos descritores: cirurgião-dentista; pneumonia associada a ventilação mecânica; prevenção, e será realizado em todos os índices, buscando captar o maior número de artigos publicados no período proposto que abordem a temática em discussão, visando o desenvolvimento fidedigno desse estudo.

Para uma análise crítica e reflexiva dos estudos incluídos na revisão, foi realizada uma leitura minuciosa e criteriosa destacando os que atingirem os critérios de inclusão e que contemplaram o objetivo proposto, para viabilizar o resultado da pesquisa de forma objetiva.

Foram inclusos artigos publicados em língua portuguesa e inglesa, nos últimos cinco anos, com texto completo, disponível on-line, com acesso livre. Foram mantidos na amostra os artigos publicados em línguas estrangeiras, que apresentarem o texto na integra.

Após as buscas através do cruzamento dos descritores nas bases indexadas: "cirurgião-dentista", "pneumonia associada a ventilação mecânica", "prevenção", foram encontradas um total de 1.147 estudo. Desses estudos um total de 358 foram encontrados na base de dados Scielo, dos quais apenas 245 apresentavam o texto completo disponível, e 67 foram publicados nos últimos cinco anos, os quais após uma análise crítica constatou-se que apenas 04 artigos respondem à questão norteadora do estudo em questão.

Ao que se refere à base de dados Lilacs, após o cruzamento dos descritores foram encontrados um total de 423 estudos, dos quais apenas 257 estudos apresentavam o texto completo e disponível, e apenas 125 estudos foram publicados nos últimos 5 anos obedecendo os critérios previamente estabelecidos, respondendo assim, após uma análise crítica 3 artigo à questão norteadora desse estudo.

Quanto à base de dados BBO foram encontrados um total de 366 estudos, dos quais apenas 153 apresentavam o texto completo disponível, e 69 foram publicados nos últimos cinco anos, os quais após uma análise crítica constatou-se que 3 artigos respondem à questão norteadora do estudo em questão.

Dessa forma, totalizaram um total de 10 estudos que iram compor esse estudo, os quais após análise crítica constatouse que respondem à questão norteadora do estudo aqui proposto, apresentados a partir do corpus desse estudo. 
Quadro 1. Corpus do Estudo.

\begin{tabular}{|c|c|c|c|c|}
\hline N\% ANO & TITULO DO ESTUDO & PERIÓDICO & AUTORES & $\begin{array}{c}\text { BASE DE } \\
\text { DADOS }\end{array}$ \\
\hline A1/ 2021 & $\begin{array}{l}\text { Aplicação do bundle para pneumonia } \\
\text { associada a ventilação mecânica em } \\
\text { neonatologia: revisão integrativa }\end{array}$ & PECIBES & $\begin{array}{l}\text { Chagas, L. L., } \\
\text { Azevedo, A. } \quad \text { M. } \quad \text { O., } \\
\text { Santos, S. C. S., } \\
\text { Vilena, K. C. } \quad \text { G., \& } \\
\text { Muller, K. T. C. }\end{array}$ & SCIELO \\
\hline A2/ 2017 & $\begin{array}{l}\text { Influence of dentistry professionals and } \\
\text { oral health assistance protocols on } \\
\text { intensive care unit nursing staff. A survey } \\
\text { study }\end{array}$ & $\begin{array}{l}\text { Rev Bras Ter } \\
\text { Intensiva. }\end{array}$ & $\begin{array}{l}\text { Blum, D. F. C., } \\
\text { Munaretto, J., } \\
\text { Baeder, F. M., } \\
\text { Gomez, J., Castro, } \\
\text { C. P. P., \& Bona, A. D. }\end{array}$ & SCIELO \\
\hline A3/ 2017 & $\begin{array}{l}\text { Bundles de prevenção de pneumonia } \\
\text { associada à ventilação } \\
\text { Mecânica: a importância da } \\
\text { multidisciplinaridade }\end{array}$ & $\begin{array}{l}\text { Revista } \\
\text { Perspectivas } \\
\text { Online: } \\
\text { Biológicas \& \& } \\
\text { Saúde }\end{array}$ & $\begin{array}{l}\text { Chicayban, L. M., } \\
\text { Terra, E. L. V. S., } \\
\text { Ribela, J. S., \& } \\
\text { Barbosa, P. F. }\end{array}$ & SCIELO \\
\hline $\mathrm{A} 4 / 2017$ & $\begin{array}{l}\text { The Insertion of Dentistry in Intensive } \\
\text { Care Units }\end{array}$ & J Health Sci & $\begin{array}{l}\text { Santosa, T. B., } \\
\text { Amaralb, M. A., } \\
\text { Peraltac, N. G., \& } \\
\text { Almeidaa, R. S. }\end{array}$ & SCIELO \\
\hline A5/ 2018 & $\begin{array}{l}\text { Avaliação odontológica de pacientes em } \\
\text { unidade de terapia intensiva (UTI) } \\
\text { pediátrica }\end{array}$ & $\begin{array}{l}\text { Rev. CES } \\
\text { Odont }\end{array}$ & $\begin{array}{l}\text { Austríaco-Leite, H.L., } \\
\text { Ferreira-Lopes, F., } \\
\text { Alves-Cardoso, M. S. S., } \\
\text { \& Diniz-Souza, L. C. }\end{array}$ & LILACS \\
\hline A6/ 2016 & $\begin{array}{l}\text { Percepção dos profissionais } \\
\text { trabalham na Unidade de Terapia } \\
\text { Intensiva (UTI) quanto à inclusão do } \\
\text { cirurgião-dentista na equipe }\end{array}$ & Full Dent. Sci. & $\begin{array}{l}\text { Lima, A.K.M.M.N., } \\
\text { Cabral, G. M. P., } \\
\text { Araújo, T. L. C., } \\
\text { Franco, M. S. P., } \\
\text { Júnior, J. L. A., \& } \\
\text { Amaral, R. C. }\end{array}$ & LILACS \\
\hline A7/2021 & $\begin{array}{l}\text { Atuação do cirurgião-dentista no cuidado } \\
\text { de pacientes em unidade de terapia } \\
\text { intensiva durante a pandemia da Covid-19 }\end{array}$ & $\begin{array}{l}\text { Brazilian Journal } \\
\text { of Health Review }\end{array}$ & $\begin{array}{l}\text { Carvalho, R. C. L., } \\
\text { Filho, R. N., Braga, R. N., } \\
\text { Silva, G. C., Marques, D. } \\
\text { M. C., \& Carvalho, } \\
\text { T.Q.A. }\end{array}$ & LILACS \\
\hline $\mathrm{A} 8 / 2020$ & $\begin{array}{l}\text { Pneumonia and the role of dentistry on } \\
\text { ICU Staff: 8-year analysis of health } \\
\text { indicators }\end{array}$ & $\begin{array}{l}\text { Brazilian Journal } \\
\text { of Health Review }\end{array}$ & $\begin{array}{l}\text { Bezerra, T. P., } \\
\text { Campêlo, C. S. P., } \\
\text { Oliveira, F. A. F., } \\
\text { Forte, C. P. F., } \\
\text { Gomes, A. K. P., } \\
\text { Silva, P. G. B., } \\
\text { Carvalho, B. M., } \\
\text { Alves, A. P. N. N., } \\
\text { \& Sousa, F. B. }\end{array}$ & B. B. O \\
\hline A9/ 2021 & $\begin{array}{l}\text { Pneumonia associated with mechanical } \\
\text { ventilation: prevention strategies used by } \\
\text { the multi- } \\
\text { professional team }\end{array}$ & $\begin{array}{l}\text { Research, Society } \\
\text { and Development }\end{array}$ & $\begin{array}{l}\text { Silva, J. F. T., } \\
\text { Brito, J. S., Alves, N. S., } \\
\text { et al. }\end{array}$ & B. B. O \\
\hline A10/ 2017 & $\begin{array}{l}\text { Contribuição do residente do serviço de } \\
\text { CTBMF na manutenção do Bundle de } \\
\text { Prevenção da PAV na UTI de um hospital } \\
\text { de trauma do Sistema Único de Saúde do } \\
\text { RS: relato de experiência. }\end{array}$ & Coleciona Sus & Flach, R. & B. B. O \\
\hline
\end{tabular}

Fonte: Autores.

\section{Resultados e Discussão}

\subsection{A prevalência e os malefícios da PAVM aos pacientes em IOT}

A PAVM está categorizada entre as IRAS mais prevalentes nas UTIs, pois apresenta dados estatísticos alarmantes de morbimortalidade e leva a danos expressivos no quadro clínico patológico do indivíduo. Dados sugerem que o paciente intubado em VM por um tempo superior a 48 horas tem de 6 a 21 vezes maior risco de desenvolver PAV (Bansal et al., 2013). 
Segundo Kiyoshi-Teo e Blegen (2015), a PAVM pode apresentar uma prevalência de até 52\% a cada 100 pacientes internados em uma UTI, esse dado pode variar dependendo dos fatores populacionais, e dos critérios utilizados na pesquisa. Ainda é citado que nos pacientes em IOT a taxa da PAVM é de até 21 vezes maior do que em outros pacientes, elevando assim, seu risco de mortalidade.

Nesse seguimento, é citado por Gibney et al., (2015), que a PAVM apresenta uma taxa de mortalidade de 24 a $50 \%$ podendo chegar a $76 \%$ se for causada por cepas multirresistentes. Ainda é citado por outros autores que sua taxa de mortalidade pode chegar à $80 \%$, ou seja, essa patologia apresenta alto risco para o paciente, causando risco de vida iminente (Saddki et al., 2017; Meng et al., 2017)

Sabe-se que a PAVM é proveniente do desequilíbrio entre o sistema imunológico orgânico e o agente microbiano causador dessa patologia, devido ao tamanho do inóculo ou do fator de virulência do patógeno, esse desequilíbrio permite a invasão deste patógeno e a propagação da doença no organismo humano (Morais et al., 2010).

Saldanha et al., (2015), cita que os pacientes intubados acumulam secreções contaminadas acima do cuff, pois perdem a barreira natural entre a orofaringe e a traqueia, eximindo assim, o reflexo da tosse. Esse processo leva a maior colonização da estrutura traqueobrônquica e a aspiração de secreções contaminadas para as vias aéreas inferiores.

Segundo Baeder et al., (2012), existem quatro possibilidades que podem levar à PAVM, sendo elas a aspiração do conteúdo orofaríngeo que fica acumulado acima do cuff, a contaminação do equipamento respiratório, que pode ocorrer no momento da intubação ou na manipulação do tubo, através da transmissão direta de uma pessoa para a outra, e pela disseminação hematogênica.

Lacerda et al., (2017), relata que a PAVM pode vir a ser adquirida também através de fatores inerentes a função pulmonar, como a partir de infecções pélvicas, feridas cirúrgicas e infecções de dispositivos como cateteres vasculares.

\subsection{Fatores de risco associados a VM}

Sabe-se que a utilização prolongada da VM nos pacientes em uso da IOT está diretamente associada a elevação da morbimortalidade nas UTI's. Estatisticamente a cada dia de permanência intubado em VM, o paciente apresenta de 1 a 3\% no aumento do risco para a ocorrência da PAVM. Os principais fatores que levar a esse aumento se dá ao uso excessivo de antibióticos, uso de técnicas invasivas e permanência prolongada de pacientes com doenças graves (Kaya et al., 2017).

É citado por Miranda et al., (2016), que além da influência do tempo prolongado de permanência na VM, há fatores relacionados a severidade da doença de base, a presença de comorbidades, acesso aos dispositivos de suporte ventilatório e a assistência à saúde empregada aos pacientes.

Quando relacionado as condições relacionadas a assistência à saúde, algumas podem se enquadrarmos fatores de risco para a PAV, como o uso de antimicrobianos, pois aumentam a colonização da orofaringe e/ou estômago por bactérias patogênicas, cita-se ainda cuidados que podem levar à aspiração para o trato respiratório ou refluxo no trato gastrointestinal, como a intubação ou reintubação orotraqueal, uso de sondas gastrointestinais, a posição da cabeceira, a imobilidade, bem como a higienização das mãos (Blevins, 2011).

Nota-se então que vários são os fatores de riscos que estão relacionados a PAVM, podendo estes serem classificados como modificáveis e não modificáveis. Sendo assim, em alguns casos os profissionais envolvidos no processo de cuidados ao paciente intubado em ventilação mecânica exerce influência direta na recuperação ou prolongamento do estado de doença do paciente (Schlesenser et al., 2012).

Dentro desta divisão, os fatores modificáveis, enfatiza-se aqueles relacionados à microbiota da UTI e a cuidados, como profilaxias, desinfecção, cuidados e protocolos para utilização de antibióticos, monitoramento das invasões e 
procedimentos. Os não modificáveis estão relacionados ao paciente, como a presença de comorbidades, idade e escore de gravidade na admissão (Rodrigues et al., 2011).

\subsection{As medidas preventivas empregadas pela equipe multidisciplinar}

A prevenção tem como objetivo prever, antecipar a emergência e o desenvolvimento de situações não desejáveis (Álvares et al., 2017). No caso da PAVM, as medidas preventivas podem diminuir a ocorrência de fatores de risco como a bronca aspiração, e os fatores modificáveis em geral, ou seja, ou que estão relacionados na maioria das vezes a assistência empregada ao paciente intubado (Cardoso et al., 2020).

Essas medidas preventivas centradas nas ações da equipe que presta assistência ao paciente sob VM são de suma importância, dentre as quais pode-se citar a higienização das mãos, a implantação de protocolos que estimulem o uso racional de antimicrobianos, a interrupção diária da sedação, o desmame ventilatório e, ainda, estratégias que visam à redução de procedimentos invasivos nas UTI's (Cabral et al., 2020).

É citado por Brito et al., (2020), que além dessas medidas empregadas, é importante também enfatizar a prevenção da colonização do trato gastrointestinal, a manutenção da cabeceira elevada a $30^{\circ}$ ou mais, a higiene oral dos pacientes pelo menos 2x ao dia, a antissepsia correta das vias aéreas e o uso de medidas assépticas para a manipulação de próteses e circuitos ventilatórios.

Nesse seguimento, percebe-se que as medidas preventivas para a PAVM são diversas e complexas, com isso, tem sido desenvolvidos e preconizado o uso de pacotes de cuidados (bundles), em vez de medidas isoladas de prevenção. Esses pacotes visam o desenvolvimento através de checklists diários com o paciente intubado (Cruz et al., 2017).

É afirmado por Fallahi et al., (2020), que medidas como elevação da cabeceira entre 30 e $45^{\circ}$, a interrupção diária da sedação, a profilaxia de úlceras pépticas, e a profilaxia de TEV são essenciais para prevenir a PAVM, e que sua realização tem que ser contínua e considerada de total importância pela equipe multidisciplinar.

Com isso, nota-se que as medidas preventivas para a redução da incidência de PAVM normalmente consistem em intervenções baseadas em protocolos e diretrizes. De modo geral, essas medidas preventivas dependem do envolvimento de toda equipe de atendimento, composta por médicos, fisioterapeutas, enfermeiros, nutricionistas, dentistas, auxiliares de enfermagem e serviços que colaboram direta ou indiretamente, de acordo com as necessidades de cada unidade (Chicayban, Terra \& Ribela, 2017).

\subsection{Os desafios enfrentados pelos odontologistas em uma UTI frente a PAVM}

Sabe-se que são diversos os desafios enfrentados pelo odontologista na prevenção da PAVM em uma UTI, e que seu papel é imprescindível nesse quesito. De acordo com Bezerra et al., (2020), dentre as contribuições do odontologista em uma UTI, a mais importante é as prevenções da PAVM, tendo em vista que esta representa $25 \%$ de todas as infecções dentro de uma UTI.

É importante frisar que recursos como a VM podem levar o paciente a vários riscos, podendo ainda os indivíduos desse grupo possuir periodontopatias, essas podem agravar ainda mais uma condição sistêmica pré-existente e influenciar o curso das infecções respiratórias, em especial, as pneumonias, sendo assim, o odontologista seria o profissional que idealizaria uma conduta direcionada a saúde bucal desse paciente, atuando inclusive nessas vertentes (Lima, Cabral \& Araújo, 2016).

É de conhecimento que a cavidade oral sofre colonização contínua, servindo de reservatório permanente de microrganismos, os quais podem ser disseminados para outras partes do organismo por meio dos procedimentos hospitalares de rotina na UTI, como através da manipulação do tubo orotraqueal (TOT), o qual pode transportar esses patógenos até os pulmões, levando a PAVM (Blum et al., (2017). 
Sendo assim, em meio a esta nova área da inserção, o odontologista deve estar qualificado para a interpretação de exames complementares, diagnosticar e prever situações indesejáveis que cerquem a cavidade oral, e elaborar junto a equipe multiprofissional, medidas preventivas para a PAVM, que apresenta elevado nível de incidência (Chicayban et al., 2017).

Nesse seguimento, o cirurgião-dentista objetiva nesse contexto a realização de exames clínicos para que possa identificar as alterações na cavidade oral, a remoção de focos infecciosos, o tratamento de lesões orais, fazendo assim, com que a terapia medicamentosa não se interrompa, não causando maiores danos a esse paciente com quadro clinico grave (Chagas et al., 2021).

Com isso percebe-se que a presença do cirurgião-dentista se torna imprescindível para a integralidade da saúde dos pacientes hospitalizados em UTI, pois estes pacientes apresentam-se em um quadro grave, e necessitam de cuidados específicos pois sua condição os torna mais susceptíveis à instalação de infecções como a PAVM (Santos et al., 2017).

Em concordância a isso, é citado por Austríaco-Leite et al., (2018), que é de extrema importância que a odontologia se integre ao atendimento dos pacientes em IOT internados nas UTI's, pois através disso se possibilita a minimização do risco de disseminação de patógenos da cavidade bucal. É importante que estes atuem na manutenção da higienização dos dentes, gengiva, bochecha e língua (Lima et al., 2016).

Em um estudo desenvolvido por Carvalho et al., (2021), em uma UTI em meio a pandemia do Covid 19, o cirurgiãodentista é citado como o profissional mais capacitado a fazer o controle de microrganismos na cavidade oral, tendo em vista que a presença de patógenos nesses casos, podem vir a causar malefícios ao sistema respiratório, levando a maior gravidade do quadro clinico do mesmo.

Além disso, a integração do cirurgião-dentista na equipe multiprofissional atuante nas UTIs leva a ampliação do campo de ensino e atuação do profissional, possibilitando a formulação e adaptação de diretrizes e bundles, pois são os profissionais mais capacitados nessa área de conhecimento (Carvalho et al., 2021; Silva et al., 2021).

\section{Considerações Finais}

A partir do desenvolvimento desse estudo de revisão de literatura, notou-se que a PAVM é uma patologia de muita incidência, apresentando uma elevada taxa de morbimortalidade entre os pacientes intubados internados em UTI's. Desta forma, seu diagnóstico, bem como a aplicação do tratamento devem ser precoces a fim de reduzir a severidade da doença e melhorar seu prognóstico.

Toda a equipe multidisciplinar em saúde alocada nas UTI's, inclusive os odontologistas, devem agir em concordância, adotando medidas de prevenção da PAVM a fim de reduzir os riscos para a sua ocorrência, sempre seguindo uma linha de cuidado integrada, diminuindo assim, a taxa de mortalidade de seus pacientes.

\section{Referências}

Alecrim, R. X., et al. (2019). Boas práticas na prevenção de pneumonia associada à ventilação mecânica. Acta Paulista de Enfermagem, $32(1)$, 11-17.

Álvares, J. F., et al. (2017). A Relevância Do Cirurgião-Dentista Na Uti: Educação, Prevenção E Mínima Intervenção. Revista Ciências e Odontologia, 1(1), $18-23$.

Austríaco-Leite, H. L., et al. (2018). Dental assessment of patients in paediatric intensive care unit (ICT). Rev. CES Odont, $31(2), 6-14$.

Baeder, F. M., et al. (2012). Condição odontológica em pacientes internados em unidade de terapia intensiva. Pesq Bras Odontop Clín Int, 12(4), 517-20.

Bansal, Marshal, et al. (2013). Potential role of periodontal infection in respiratory diseases - a review. J Med Life, 6(3), 244-8.

Barcellos, R. A., et al. (2020). Impacto de uma lista de verificação multiprofissional nos tempos de ventilação mecânica invasiva e de permanência em UTI. Jornal Brasileiro de Pneumologia, 46(3).

Bezerra, T. P., Campêlo, C. S. P., Oliveira, F. A. F., et al. (2020). Pneumonia and the role of dentistry on ICU Staff: 8-year analysis of health indicators. Brasilian Journal of ciences. 19. 
Blevins, J. Y. (2011). Oral Health Care For Hospitalized Children. Ped Nursing. Septem-ber-October, 37(5).

Blum, D. F. C., et al. (2017). Influence of dentistry professionals and oral health assistance protocols on intensive care unit nursing staff. A survey study. Rev Bras Ter Intensiva, 29(3), 391-393.

Branco, A., et al. (2020). Educação para prevenção da pneumonia à ventilação mecânica em unidade de terapia intensiva. Rev Bras Enf., 73(6).

Brito, A., et al. (2020). Pneumonia associated with mechanical ventilation: consequences and mortality in an intensive therapy unit. Electronic Journal Collection Health, 44, 1-9.

Cabral, B. G., et al. (2020). Cuidados Preventivos Para Pneumonia Associada A Ventilação Mecânica: Revisão Integrativa Preventive Care For Pneumonia Mechanical Ventilation Associated: Integrative Review. Revista enfermagem atual in derme, 131-140.

Cardoso, T. F., et al. (2020). COVID-19 e a Cavidade Bucal: Interações, Manifestações Clínicas e Prevenção. Journal of medicine, 1 , $98-105$.

Carvalho, R. C. L., et al. (2021). Atuação do cirurgião-dentista no cuidado de pacientes em unidade de terapia intensiva durante a pandemia da Covid-19. Brazilian Journal of Health Review, Curitiba, 4(2), 9473-9487.

Chagas, L. L., et al. (2021). Aplicação do bundle para pneumonia associada a ventilação mecânica em neonatologia: revisão integrativa. PECIBES, 1, 18-76.

Chicayban, L. M., et al. (2017). Bundles de prevenção de pneumonia associada à ventilação mecânica: a importância da multidisciplinaridade. Revista Perspectivas Online: Biológicas \& Saúde, 7(25), 25- 35.

Chicayban, L. M., Terra, E. L. V. S., \& Ribela, J. S. (2017). Bundles de prevenção de pneumonia associada à ventilação mecânica: a importância da multidisciplinaridade. Revista Perspectivas Online: Biológicas \& Saúde Novembro, 7(25), 25- 35.

Cruz, S. M. Q., et al. (2017). Microbiota de los ecosistemas de la cavidad bucal. Revista Cubana de Estomatologia, 54(1), 84-99, 2017.

Fallahi, H. R. et al. (2020). Being a front-line dentist during the Covid- 19 pandemic: aliterature review. 5.

Furtado, M. V. C., et al. (2020). Abordagem multiprofissional na pneumonia associada à ventilação mecânica. Rev. Elet. Acervo Saúde., 12(10), 1-10.

Gibney, J., et al. (2015). Nurses' knowledge, attitudes, and current practice of daily oral hygiene care to patients on acute aged care wards in two Australian hospitals. Spec Care Dentist, 35(6), 285-93.

Kaya, H.; et al. (2017). Effects of oral care with glutamine in preventing ventilator-associated pneumonia in neurosurgical intensive care unit patients. Appl Nurs Res, 33, 10-4.

Kiyoshi-Teo, H., et al. (2015). Influence of Institutional Guidelines on Oral Hygiene Practices in Intensive Care Units. Am J Crit Care. 24(4), 309-18.

Lacerda, V. C. F., et al. (2017). Impact of oral hygiene involving toothbrushing versus chlorhexidine in the prevention of ventilator-associated pneumonia: a randomized study. BMC Infect Dis, 17(1):112.

Lima, A. K. M. M. N., Cabral, G. M. P., \& Araújo, T. L. P. (2016). Percepção dos profissionais que trabalham na Unidade de Terapia Intensiva (UTI) quanto à inclusão do cirurgião-dentista na equipe. Full Dent. Sci. 7(28),72-75.

Lima, A. K. M. M. N., et al. (2016). Percepção dos profissionais que trabalham na Unidade de Terapia Intensiva (UTI) quanto à inclusão do cirurgião-dentista na equipe. Full Dent. Sci. 7(28):72-75.

Mendes, K. D. S., Silveira, R. C. C. P., \& Galvão, C. M. (2008). Integrative literature review: a research method to incorporate evidence in health care and nursing. Reflexão Texto Contexto - Enferm, 17 (4).

Meng, L., et al. (2016). Early vs late tracheostomy in Critically ill Patients: a systematic review and meta-analysis. Clin Respir J, 10, 684-692

Miranda, A. F., et al. (2016). Oral care practices for patients in Intensive Care Units: a pilot survey. Indian J Crit Care Med, 20(5), $267-73$.

Morais, T. M. N., et al. (2010). A importância da atuação odontológica em pacientes internados em unidade de terapia intensiva. Rev Bras Ter Int, 18(4), 4127.

Oliveira, J. G. O., et al. (2021). Prevenção de pneumonia associada a ventilação mecânica: uma revisão integrativa. Rev. Saúde em Foco, 1(13), 211-222. 2021.

Pinto, A. C. S., et al. (2021). Efficiency of different protocols for oral hygiene combined with the use of chlorhexidine in the prevention of ventilatorassociated pneumonia. Jornal Brasileiro de Pneumologia, 47(1).

Rodrigues, V. P., et al. (2011). Avaliação dos hábitos de higiene bucal de crianças durante período de internação hospitalar. Odontol. Clín.-Cient. (Online), $10(1)$.

Saddki, N., et al. (2017). Oral care for intubated patients: a survey of intensive care unit nurses. Nurs Crit Care, 22(2), 89-98.

Saldanha, K. F. D., et al. (2015). A odontologia hospitalar: revisão. Arch Health Invest, 4(1), 58-68.

Santos, C., et al. (2020). Boas práticas de enfermagem a pacientes em ventilação mecânica invasiva na emergência hospitalara. Escola Anna Nery, 24(2), e20190300. 2020.

Santos, T. B., et al. (2017). A Inserção da Odontologia em Unidades de Terapia Intensiva. J Health Sci, 19(2), 83-8. 
Research, Society and Development, v. 10, n. 13, e378101321426, 2021

(CC BY 4.0) | ISSN 2525-3409 | DOI: http://dx.doi.org/10.33448/rsd-v10i13.21426

Schlesener V. F., et al. (2012). O cuidado com a saúde bucal de pacientes em UTI. Cinergis. 13(1),73-77.

Silva, J. F. T., et al. (2021). Pneumonia associada a ventilação mecânica: estratégias de prevenção utilizadas pela equipe multiprofissional. Research, Society and Development, $10,9$.

Silva, T. C., et al. (2021). Conhecimento de enfermagem em paciente adulto com pneumonia associada a ventilação mecânica (PAVM): uma revisão integrativa. Bra Jou of Dev, 7(6), 57384-57391.

Souza, M. T., Silva, M. D., \& Carvalho, R. (2010). Revisão integrativa: o que é e como fazer. Einstein. 8(1 Pt 1), 102-6. 